\title{
A $o(n)$ MONOTONICITY TESTER FOR BOOLEAN FUNCTIONS OVER THE HYPERCUBE
}

\author{
D. CHAKRABARTY* AND C. SESHADHRI ${ }^{\dagger}$
}

\begin{abstract}
A Boolean function $f:\{0,1\}^{n} \mapsto\{0,1\}$ is said to be $\varepsilon$-far from monotone if $f$ needs to be modified in at least $\varepsilon$-fraction of the points to make it monotone. We design a randomized tester that is given oracle access to $f$ and an input parameter $\varepsilon>0$, and has the following guarantee: It outputs Yes if the function is monotonically non-decreasing, and outputs No with probability $>2 / 3$, if the function is $\varepsilon$-far from monotone. This non-adaptive, one-sided tester makes $O\left(n^{7 / 8} \varepsilon^{-3 / 2} \ln (1 / \varepsilon)\right)$ queries to the oracle.
\end{abstract}

1. Introduction. Testing monotonicity of Boolean functions is a classical question in property testing. The Boolean hypercube $\{0,1\}^{n}$ defines a natural partial order with $x \prec y$ iff $x_{i} \leq y_{i}$ for all $i \in[n]$. A Boolean function $f:\{0,1\}^{n} \mapsto\{0,1\}$ is monotone if $f(x) \leq f(y)$ whenever $x \prec y$.

A Boolean function's distance to monotonicity is the minimum fraction of points at which it needs to be modified to make it monotone. In the property testing framework we are provided oracle access to the function $f$ and are given a parameter $\varepsilon>0$. A monotonicity tester is an algorithm that accepts if the function is monotone, and rejects if the function is $\varepsilon$-far from monotone. The tester is allowed to be randomized, and has to be correct with non-trivial probability (say $>2 / 3$ ). The tester is called one-sided if the tester always accepts a monotone function. The tester is non-adaptive if the queries made by the algorithm do not depend on the answers given by the oracle.

The quality of a monotonicity tester is governed by the number of oracle queries as well as the running time. Goldreich et al. [8] suggested the following simple tester: query the function value on a pair of points that differ on exactly a single coordinate and reject if monotonicity is violated. In other words, the tester samples a random edge of the hypercube and checks for monotonicity between the two endpoints. This is called the edge tester for monotonicity. It is clear the running time is of the same order as the number of queries

Goldreich et al. [8] show that $O(n / \varepsilon)$-queries by the edge tester suffice to test monotonicity. Their analysis is tight, so the edge tester can do no better. They explicitly ask whether there exists a tester with an improved query complexity in terms of $n$. Fischer et al. [7] show that any non-adaptive, one-sided tester ${ }^{1}$ for monotonicity must make $\Omega(\sqrt{n})$-queries for constant $\varepsilon>0$. While monotonicity has been extensively studied in property testing $[6,8,5,11,7,9,13,1,3,2,4]$, no significant progress had been made on this decade old question of testing monotonicity of Boolean functions. Our main result is an affirmative answer to the above question of [8].

THEOREM 1.1. There exists a one-sided, non-adaptive $O\left(n^{7 / 8} \varepsilon^{-3 / 2} \ln ^{-1}(1 / \varepsilon)\right)$-query monotonicity tester for Boolean functions $f:\{0,1\}^{n} \mapsto\{0,1\}$.

\footnotetext{
*Microsoft Research, 9 Lavelle Road, Bangalore 560001, India. dechakr@microsoft.com

†Sandia National Labs, Livermore, USA. scomand@sandia.gov

${ }^{1}[7]$ also show a $\Omega(\log n)$ lower bound for 2-sided testers.
} 
We get an improved bound for functions with low average sensitivity. Given a Boolean function $f$, the influence of dimension $i$ is the fraction of edges of the hypercube crossing the $i$ th dimension whose endpoints have different function values. The average sensitivity, denoted as $\mathbf{I}(f)$, is the sum of all the $n$ influences. The functions defined in [7] to prove the lower bound of $\Omega(\sqrt{n})$ for non-adaptive, one-sided testers have constant average sensitivity, and hence the following is optimal for such functions.

THEOREM 1.2. There exists a one-sided, non-adaptive $O\left(n^{1 / 2} \varepsilon^{-6} \mathbf{I}^{3}(f) \ln (1 / \varepsilon)\right)$-query monotonicity tester for Boolean functions of average sensitivity $\mathbf{I}(f)$.

Remark 1 (Pair testers). A pair tester [5] describes a fixed distribution (independent of the function) on domain pairs $(x \prec y)$, makes independent queries on pairs drawn from this distribution, and rejects iff some drawn pair violates monotonicity. By definition, pair testers are non-adaptive and one-sided. (Note that the edge tester is a pair tester.) Briët et al. [3] show that any pair tester with a linear dependence of $\varepsilon^{-1}$ must make $\Omega(n /(\varepsilon \log n))$ queries. The linear dependence is crucial in their argument. Our tester is also a pair tester. We circumvent the lower bound (on n) of [3] because of the worse dependence on $\varepsilon$.

1.1. Main Ideas. Our tester is a combination of the edge tester and what we call the path tester. The path tester essentially does the following. It samples a random point $x$ on the hypercube, performs a sufficiently long random length walk on the directed hypercube to reach $y$, queries $f(x)$ and $f(y)$ and tests for monotonicity. We stress that the path tester does not query all the points along the path, but just the end points.

Our algorithm is inspired by a recent paper by Ron et al. [14], which shows a $O(\sqrt{n})$ query randomized algorithm to estimate the average sensitivity of a monotone function. The algorithm essentially performs the operation above and counts the number of mismatches; Ron et al. [14] explicitly ask whether an algorithm "in the spirit" above can be used for monotonicity. Our answer is yes.

Consider a function $f$ which is $\varepsilon$-far from monotone. The aim of any tester is to detect a violation, that is, a pair $x \prec y$ such that $1=f(x)>f(y)=0$. The success probability of the edge tester is exactly the fraction of violated edges. The intuition is that there are possibly many more violations that are "far away" and the directed random walk will help detect those. Consider the function $f:\{0,1\}^{n+1} \mapsto\{0,1\}$, $f(0, x)=0$ if $|x| \leq n / 2-2 \sqrt{n}$ and 1 otherwise; $f(1, x)=0$ if $|x| \leq n / 2+2 \sqrt{n}$ and 1 otherwise. Here $|x|$ is the number of $1 \mathrm{~s}$ in $x$. This function has a constant distance to monotonicity, and all the violated edges are of the form $((0, x),(1, x))$ for $n / 2-2 \sqrt{n} \leq|x| \leq n / 2+2 \sqrt{n}$. The edge tester detects a violation with probability only $\Theta(1 / n)$. Suppose we pick a uniform random point and perform a random walk of length $\sqrt{n} / 2 \leq \ell<\sqrt{n}$. If the starting point has 0 in the first coordinate and any of the $\Theta(\sqrt{n})$ steps flips the first coordinate, the end points of the walk exhibit a violation to monotonicity. This happens with probability $\Theta(1 / \sqrt{n})$, handily beating the edge tester.

The argument above required the violated edges to be aligned along one dimension. In $\S 2.2$, we prove that a directed random walk detects a violation with sufficiently high probability when there is a large matching of violated edges. One of the ingredients of this proof is the following interesting combinatorial observation. In $\S 2.1$, we prove that if a $\sigma$-fraction of the hypercube is marked blue, then the probability that the 
random walk starts and ends at a blue point is $\widetilde{\Omega}\left(\sigma^{2}\right)$. It shows that the endpoints of this random walk, which are highly correlated, behave like two independent samples as far as being blue is concerned.

But what if no large matching of violated edges exists? Take the 'anti-majority' function defined as $f(x)=1$ if $|x| \leq n / 2$, and $f(x)=0$ otherwise. This function is $1 / 2$-far from monotone, and yet the largest matching of violated edges is of size $\Theta\left(2^{n} / \sqrt{n}\right)$. This is dealt with by our dichotomy theorem. In $\S 2.3$, we prove that for any $s>0$, either there exists a set of $\Theta\left(s \varepsilon 2^{n}\right)$ violated edges, or there exists a matching of $\Theta\left(\varepsilon 2^{n} / s\right)$ violated edges. With this we are done; in the former case, the edge tester suffices, in the latter the path tester suffices.

The proof of our dichotomy theorem combines two ideas discovered earlier in the context of monotonicity testing. The first is a theorem of Lehman and Ron [11] on multiple source-sink routing over the hypercube. The second is an alternating paths machinery developed by the authors in a separate work [4] on general range monotonicity testing.

1.2. Isoperimetry for the directed hypercube. The problem of Boolean monotonicity testing is intimately connected with isoperimetric questions on the $d i$ rected hypercube. We use $E$ for the set of undirected edges of the hypercube and $E(S, T)$ for the set of undirected edges from $S$ to $T$. Similarly, we use $E^{+}$and $E^{+}(S, T)$ to denote the directed versions.

Any function $f:\{0,1\}^{n} \mapsto\{0,1\}$ can be thought of as an indicator for the subset $S=\{x \mid f(x)=1\}$. We use $\mu$ to denote $|S| / 2^{n}$, the uniform measure of $S$. Let $\Phi(S)$ be the total influence of $S$, which is $|E(S, \bar{S})| / 2^{n-1}$. Let $\partial(S)$ be the boundary of $S$, that is, $\{x \mid(x, y) \in E, x \in S, y \notin S\}$. The standard edge isoperimetric bound for the undirected hypercube states that $\Phi(S) \geq 2 \mu$, whenever $\mu \leq 1 / 2$. Harper's theorem [10] proves that $|\partial(S)|$ is minimized when $S$ is a Hamming ball. Margulis [12] proves the remarkable fact that both $\Phi(S)$ and $\partial(S)$ cannot be minimized simultaneously. Formally, he proves that $\Phi(S) \cdot|\partial(S)|=\Omega\left(\mu^{2}\right)$, whenever $\mu \leq 1 / 2$. (This is actually proven for the general $p$-biased measures.)

What about the directed hypercube? We can define $\Phi^{+}(S)=\left|E^{+}(S, \bar{S})\right| / 2^{n-1}$ and $\partial^{+}(S)=\left\{x \mid(x, y) \in E^{+}, x \in S, y \notin S\right\}$. Let $\varepsilon_{f}$ denote the distance of $f$ to monotonicity. The success probability of the edge tester is precisely $\Phi^{+}(S) / n$, and the classic theorem of Goldreich et al. [8] proves that $\Phi^{+}(S)=\Omega\left(\varepsilon_{f}\right)$. Our dichotomy theorem is really a directed version of Margulis' theorem. We prove that $\Phi^{+}(S) \cdot\left|\partial^{+}(S)\right|=\Omega\left(\varepsilon_{f}^{2}\right)$. It is interesting to note how $\varepsilon_{f}$ takes the place of $\mu$ in the undirected bounds.

2. The Tester and its Analysis. We start by setting some notation. For binary vectors $x, y \in\{0,1\}^{n},|x|$ is the number of 1 's in $x$ and $\|x-y\|_{1}$ is the $\ell_{1}$ distance between $x$ and $y$. The all zeros and all ones vectors are denoted $0^{n}$ and $1^{n}$, respectively. The directed hypercube is the directed graph with vertex set $\{0,1\}^{n}$, and a directed edge from $x$ to $y$ if $x \prec y$ and $\|y-x\|_{1}=1$. Throughout the paper, u.a.r. stands for 'uniformly at random'.

Our tester is given as input a parameter $\varepsilon>0$ and query access to $f$. The tester will accept if $f$ is monotone, and reject with probability $>2 / 3$ if $f$ is $\varepsilon$-far from being monotone. We assume without loss of generality that $\varepsilon \leq 1 / 2$ since any function can be made monotone by changing at most $1 / 2$ of its values. Furthermore, we will 
assume $\varepsilon \geq n^{-1 / 4}$, as conversely, Theorem 1.1 holds true by dint of the edge tester itself.

We set the following parameters. Let $C_{\varepsilon}=\sqrt{10 \ln (1 / \varepsilon)}$. By the assumptions on $\varepsilon$, we get $2<C_{\varepsilon} \leq 2 \sqrt{\ln n}$. Let $\ell:=2\left\lceil C_{\varepsilon} \sqrt{n}\right\rceil$. Note that $\ell>4 \sqrt{n}$, and $\frac{\ell}{C_{\varepsilon}}=\Theta(\sqrt{n})$. We let $I_{\ell}$ denote the index set $[n / 2-\ell / 2, n / 2+\ell / 2]$. For $1 \leq i \leq n, L_{i}:=\left\{x \in\{0,1\}^{n}\right.$ : $|x|=i\}$ denotes the $i$ th layer of the directed hypercube. We refer to $\bigcup_{i \in I_{\ell}} L_{i}$ as the middle layers of the hypercube. We say an edge of the hypercube lies in the middle layers if both its endpoints lie in the middle layers.

We now describe the random walk based procedure called the path tester. This uses a parameter $\sigma \in(0,1)$ that determines the distance between samples.

path-tester $(\sigma)$.

1. Let $\mathcal{P}$ be the collection of paths in the directed hypercube from $0^{n}$ to $1^{n}$. Pick a path $\mathbf{p} \in \mathcal{P}$ u.a.r. Let $X_{\mathbf{p}}:=\left\{z \in \mathbf{p}:|z| \in I_{\ell}\right\}$.

2. Sample $x \in X_{\mathbf{p}}$ u.a.r.

3. Let $Y_{\mathbf{p}}(x):=\left\{z \in X_{\mathbf{p}}:\|z-x\|_{1} \geq \frac{\sigma \ell}{32 C_{\varepsilon}}-1\right\}$. Sample $y \in Y_{\mathbf{p}}(x)$ u.a.r.

4. Reject if $(x, y)$ violates monotonicity; i.e. $f(x)<f(y), x \succ y$ or $f(x)>$ $f(y), x \prec y$.

This is clearly a pair tester (and is hence non-adaptive and one-sided). Our final tester runs either the path tester with a particular $\sigma$ to be fixed later, or the edge tester, each with probability $1 / 2$.

The challenge lies in lower bounding the probability of rejection when the function $f$ is $\varepsilon$-far from monotonicity. Henceforth, we assume the function $f$ is $\varepsilon$-far, and we call the rejection event a success. Since $f$ is $\varepsilon$-far from monotonicity, any maximal set $M$ of disjoint, violating pairs satisfies $|M| \geq \varepsilon 2^{n-1}$ (Lemma 3 of [7]). We refer to $M$ as a matching of violated pairs.

We start with an easy proposition.

Proposition 2.1. (a) $\left|\bigcup_{i \notin I_{\ell}} L_{i}\right| \leq \varepsilon^{5} 2^{n}$. (b) For all $i \leq n$, a u.a.r path $\mathbf{p}$ contains a u.a.r vertex from $L_{i}$.

Proof. By Chernoff bounds, for a u.a.r $x \in\{0,1\}^{n}, \operatorname{Pr}[|| x|-n / 2|>\ell / 2] \leq 2 e^{-\ell^{2} / 2 n}$. Since $\ell=2\lceil\sqrt{10 n \ln (1 / \varepsilon)}\rceil$, this probability is at most $\varepsilon^{5}$. For the second part, observe that the number of paths in $\mathcal{P}$ that pass through a given vertex $x$ depends solely on $|x|$.

2.1. Going from blue to blue. Suppose at least $\sigma 2^{n}$ vertices of the middle layers are colored blue. Let $(x, y)$ be a random pair sampled by path-tester $(\sigma)$, and let $\mathcal{E}$ be the event that both $x$ and $y$ are blue. If $x$ and $y$ were chosen independently u.a.r., then the probability of both being blue is $\sigma^{2}$. The following lemma shows that this probability does not degrade much even though $x$ and $y$ are correlated (for instance, they form an ancestor-descendant pair).

Lemma 2.2. $\operatorname{Pr}[\mathcal{E}]=\Omega\left(\frac{\sigma^{2}}{\ln (1 / \varepsilon)}\right)$. 
Proof. For notational convenience, set $\mu:=\sigma / 16 C_{\varepsilon}$. This implies ${ }^{2}\left|X_{\mathbf{p}}\right|-\left|Y_{\mathbf{p}}(x)\right| \leq \mu \ell$ for any $x \in \mathbf{p}$. Let $b(\mathbf{p})$ be the random variable denoting the number of blue points in $X_{\mathbf{p}}$ corresponding to a random path $\mathbf{p}$. Let $\mathcal{E}_{x}$ and $\mathcal{E}_{y}$ be the events that the first and second points are blue; that is $\mathcal{E}=\mathcal{E}_{x} \wedge \mathcal{E}_{y}$. Abusing notation, $\mathbf{p}$ will also denote the event that $\mathbf{p}$ is the sampled path.

Conditioned on a path $\mathbf{p}$ being sampled, the probability of the first point $x$ sampled by the path tester being blue is $b(\mathbf{p}) / \ell$. Formally, $\operatorname{Pr}\left[\mathcal{E}_{x} \mid \mathbf{p}\right]=b(\mathbf{p}) / \ell$.

Conditioned on the path being $\mathbf{p}$ and the first point being $x$ (irrespective of it being blue or not), the probability that the second point $y$ is blue is the number of blue points in $Y_{\mathbf{p}}(x)$ divided by $\left|Y_{\mathbf{p}}(x)\right|$. The number of blue points in $Y_{\mathbf{p}}(x)$ is at least $b(\mathbf{p})-\mu \ell$ since $\left|X_{\mathbf{p}}\right|-\left|Y_{\mathbf{p}}(x)\right| \leq \mu \ell$. Therefore,

$$
\operatorname{Pr}\left[\mathcal{E}_{y} \mid \mathbf{p}, x \text { first point }\right]=\frac{\mid \text { blue points in } Y_{\mathbf{p}}(x) \mid}{\left|Y_{\mathbf{p}}(x)\right|} \geq \frac{b(\mathbf{p})-\mu \ell}{\ell} .
$$

Since the above inequality holds for all $x$ (in particular, any blue $x$ ),

$$
\operatorname{Pr}\left[\mathcal{E}_{y} \mid \mathbf{p}, \mathcal{E}_{x}\right] \geq \frac{b(\mathbf{p})-\mu \ell}{\ell} .
$$

Together, we get

$$
\begin{aligned}
\operatorname{Pr}[\mathcal{E}] & =\sum_{\mathbf{p} \in \mathcal{P}} \operatorname{Pr}\left[\mathcal{E}_{y} \mid \mathbf{p}, \mathcal{E}_{x}\right] \cdot \operatorname{Pr}\left[\mathcal{E}_{x} \mid \mathbf{p}\right] \cdot \operatorname{Pr}[\mathbf{p}] \\
& \geq \sum_{\mathbf{p} \in \mathcal{P}}\left(\frac{b(\mathbf{p})-\mu \ell}{\ell} \cdot \frac{b(\mathbf{p})}{\ell} \cdot \frac{1}{|\mathcal{P}|}\right) \\
& =\frac{1}{|\mathcal{P}|} \sum_{\mathbf{p} \in \mathcal{P}}\left(\frac{b(\mathbf{p})}{\ell}\right)^{2}-\frac{\mu}{|\mathcal{P}|} \sum_{\mathbf{p} \in \mathcal{P}} \frac{b(\mathbf{p})}{\ell}
\end{aligned}
$$

In the following, we use $\mathbf{E}[\ldots]$ to denote the expectation over the choice of the path p. Note that $\mathbf{E}[b(\mathbf{p}) / \ell]:=\frac{1}{|\mathcal{P}|} \sum_{\mathbf{p} \in \mathcal{P}}(b(\mathbf{p}) / \ell)$, anf thus we can express the bound of (2.1) in terms of expectations. The second inequality is an application of Jensen's inequality.

$$
\begin{aligned}
\operatorname{Pr}[\mathcal{E}] & \geq \mathbf{E}\left[(b(\mathbf{p}) / \ell)^{2}\right]-\mu \mathbf{E}[b(\mathbf{p}) / \ell] \\
& \geq(\mathbf{E}[b(\mathbf{p}) / \ell])^{2}-\mu \mathbf{E}[b(\mathbf{p}) / \ell]=\mathbf{E}[b(\mathbf{p}) / \ell](\mathbf{E}[b(\mathbf{p}) / \ell]-\mu)
\end{aligned}
$$

The following claim lower bounds the expectation

Claim 2.2.1. $\mathbf{E}[b(\mathbf{p}) / \ell] \geq \frac{\sigma}{4 C_{\varepsilon}}$.

Proof. Note that, for all $i,\left|L_{i}\right| \leq\left(\begin{array}{c}n \\ n / 2\end{array}\right) \leq \frac{2^{n}}{\sqrt{n}}$. Let $n_{i}$ be the number of blue vertices in layer $L_{i}$. Note that $\sum_{i \in I_{\ell}} n_{i} \geq \sigma 2^{n}$. Let $Z_{i}$ be the indicator variable for the $i$ th

\footnotetext{
${ }^{2}$ The curious reader may be wonder why we have a " -1 " in the distance condition for $Y_{\mathbf{p}}(x)$ in the description of the path tester. This is a technicality so that we have the bound $\left|X_{\mathbf{p}}\right|-\left|Y_{\mathbf{p}}(x)\right| \leq \mu \ell$. Without the -1 , the bound would be $\mu \ell+2$ and can be made $O(\mu \ell)$ only for large enough $\sigma$. So instead of enforcing such a condition or carrying around a +2 , the " -1 " allows for a cleaner presentation.
} 
layer vertex in $\mathbf{p}$ being blue. Hence, $b(\mathbf{p})=\sum_{i \in I_{\ell}} Z_{i}$. For all $i$, a $\mathbf{p}$ chosen u.a.r from $\mathcal{P}$ contains a uniform random vertex in layer $L_{i}$ (Prop. 2.1). Thus,

$$
\mathbf{E}\left[Z_{i}\right]=\frac{n_{i}}{\left|L_{i}\right|} \geq \frac{\sqrt{n}}{2^{n}} \cdot n_{i}
$$

Using linearity of expectation and the bound $\ell<4 C_{\varepsilon} \sqrt{n}$,

$$
\mathbf{E}[b(\mathbf{p}) / \ell] \geq \frac{\sqrt{n}}{\ell 2^{n}} \sum_{i \in I_{\ell}} n_{i} \geq \frac{\sigma \sqrt{n}}{\ell} \geq \frac{\sigma}{4 C_{\varepsilon}} .
$$

The function $h(x)=x(x-\mu)$ is increasing when $x \geq \mu / 2$. The lower bound of Claim 2.2.1 gives $\mathbf{E}[b(\mathbf{p}) / \ell] \geq \sigma / 4 C_{\varepsilon}>\mu$. Substituting in (2.2) gives $\operatorname{Pr}[\mathcal{E}] \geq$ $\left(\sigma / 4 C_{\varepsilon}\right)\left(\sigma / 4 C_{\varepsilon}-\mu\right)$. Plugging back $\mu=\sigma / 16 C_{\varepsilon}$ completes the proof of Lemma 2.2.

2.2. Large violated-edge matchings are good. We bound the success of the path tester when a large matching of violated edges exists.

Lemma 2.3. Suppose there exists a matching $E$ of violated edges all lying in the middle layers of the hypercube. Set $\sigma=|E| / 2^{n}$. Then path-tester $(\sigma)$ succeeds with probability $\Omega\left(\frac{\sigma^{3}}{\sqrt{n} \ln (1 / \varepsilon)}\right)$.

Proof. We begin with some notation. Let the set of endpoints of edges in $E$ be $B$. We partition $B$ into $B_{0}$ and $B_{1}$, indexed by the value of the function on these vertices. That is, $B_{0}=\{x \in B: f(x)=0\}$ and $B_{1}=\{x \in B: f(x)=1\}$. Note that $\left|B_{0}\right|=\left|B_{1}\right|=|E|$. For any two points $x, y$, let $\mathcal{E}_{x, y}$ denote the event that the path tester picks $(x, y)$. For convenience, in what follows, the pairs of $E$ will be ordered according to the directed hypercube (so if $\left(z, z^{\prime}\right) \in E$, then $z \prec z^{\prime}$ ). We abuse notation to define $E$ as a function. That is, for edge $\left(z, z^{\prime}\right) \in E$, we set $E(z)=z^{\prime}$ and $E\left(z^{\prime}\right)=z$.

We define the following sets of pairs of vertices.

$$
\begin{aligned}
\Pi & =\left\{(x, y) \mid x \prec y,\|x-y\|_{1} \geq \frac{\sigma \ell}{32 C_{\varepsilon}}-1, x \in B_{1}, y \in B_{1}\right\} . \\
\Pi^{\prime} & =\{(x, E(y)) \mid(x, y) \in \Pi\} .
\end{aligned}
$$

A few observations. $\Pi$ lies in the support of the pair tester, that is, pairs $(x, y)$ sampled with non-zero probability. Every pair in $\Pi^{\prime}$ is a violation; for $(x, y) \in \Pi$, we have $x \prec y, y \in B_{1}$ implying $E(y) \succ y$ and $E(y) \in B_{0}$. Finally, the mapping $(x, y) \in \Pi$ to $(x, E(y)) \in \Pi^{\prime}$ is one-to-one. This uses the fact that $E$ is a matching (and is a crucial piece of the proof).

Since all pairs in $\Pi^{\prime}$ are violations,

$$
\operatorname{Pr}[\text { success }] \geq \sum_{\left(x, y^{\prime}\right) \in \Pi^{\prime}} \operatorname{Pr}\left[\mathcal{E}_{x, y^{\prime}}\right]
$$


Using the mapping between $\Pi^{\prime}$ and $\Pi$ and that $\operatorname{Pr}\left[\mathcal{E}_{x, y}\right]>0$ for $(x, y) \in \Pi$,

$$
\begin{aligned}
\sum_{\left(x, y^{\prime}\right) \in \Pi^{\prime}} \operatorname{Pr}\left[\mathcal{E}_{x, y^{\prime}}\right] & =\sum_{\left(x, y^{\prime}\right) \in \Pi^{\prime}} \operatorname{Pr}\left[\mathcal{E}_{x, E\left(y^{\prime}\right)}\right] \cdot \frac{\operatorname{Pr}\left[\mathcal{E}_{x, y^{\prime}}\right]}{\operatorname{Pr}\left[\mathcal{E}_{x, E\left(y^{\prime}\right)}\right]} \\
& =\sum_{(x, y) \in \Pi} \operatorname{Pr}\left[\mathcal{E}_{x, y}\right] \cdot \frac{\operatorname{Pr}\left[\mathcal{E}_{x, E(y)}\right]}{\operatorname{Pr}\left[\mathcal{E}_{x, y}\right]}
\end{aligned}
$$

We break the remaining proof into simpler claims. For a vertex $x$, define $s(x):=$ $\left|Y_{\mathbf{p}}(x)\right|=\left|\left\{z \in X_{\mathbf{p}}:\|z-x\|_{1} \geq \sigma \ell / 32 C_{\varepsilon}-1\right\}\right|$ where $\mathbf{p}$ is some path containing $x$. This is well-defined since $\left|Y_{\mathbf{p}}(x)\right|$ is independent of $\mathbf{p}$ for any $\mathbf{p} \ni x$. In fact,

$$
s(x)=\left|\left\{i \in I_{\ell}:|i-| x|| \geq \frac{\sigma \ell}{32 C_{\varepsilon}}-1\right\}\right|
$$

The following claim is a routine calculation.

Claim 2.3.1. Suppose $x, y$ are in the middle layers and $\|x-y\|_{1} \geq \frac{\sigma \ell}{32 C_{\varepsilon}}-1$. Let $\mathcal{P}_{x, y}$ denote the set of paths containing both $x$ and $y$. Define

$$
\theta_{x, y}:=\frac{1}{\ell}\left(\frac{1}{s(x)}+\frac{1}{s(y)}\right)
$$

Then,

$$
\operatorname{Pr}\left[\mathcal{E}_{x, y}\right]=\theta_{x, y} \frac{\left|\mathcal{P}_{x, y}\right|}{|\mathcal{P}|}
$$

Proof. Note that

$$
\operatorname{Pr}\left[\mathcal{E}_{x, y}\right]=\sum_{\mathbf{p} \in \mathcal{P}_{x, y}} \operatorname{Pr}[\mathbf{p} \text { sampled }] \cdot \operatorname{Pr}[x, y \text { sampled } \mid \mathbf{p} \text { sampled }] .
$$

Since $\|x-y\|_{1} \geq \frac{\sigma \ell}{32 C_{\varepsilon}}-1, y \in Y_{\mathbf{p}}(x)$ (and vice versa). Suppose $x$ is the first point to be sampled; this happens with probability $1 / \ell$. The probability that $y$ is the second point sampled is $\frac{1}{\left|Y_{\mathbf{p}}(x)\right|}$. Arguing analogously when $y$ is sampled first, when $x, y \in X_{\mathbf{p}}$,

$$
\operatorname{Pr}[x, y \text { sampled } \mid \mathbf{p} \text { sampled }]=\frac{1}{\ell}\left(\frac{1}{\left|Y_{\mathbf{p}}(x)\right|}+\frac{1}{\left|Y_{\mathbf{p}}(y)\right|}\right)=\theta_{x, y} .
$$

The proof concludes by noting that $\sum_{\mathbf{p}: x, y \in \mathbf{p}} \operatorname{Pr}[\mathbf{p}$ sampled $]=\frac{\left|\mathcal{P}_{x, y}\right|}{|\mathcal{P}|}$. $\mathbf{}$

The next claim shows that for any $(x, y) \in \Pi, \theta_{x, E(y)}$ is almost as large as $\theta_{x, y}$.

Claim 2.3.2. For $(x, y) \in \Pi, \theta_{x, E(y)} \geq \theta_{x, y} / 2$

Proof. For convenience, let $y^{\prime}$ denote $E(y)$; note that $y^{\prime} \succ y$ and $\left|y^{\prime}\right|=|y|+1$. There exists some path containing $x, y$, and $y^{\prime}$. From $(2.4), s(y) \leq s\left(y^{\prime}\right) \leq s(y)+1$.

Putting it all together,

$$
\frac{\theta_{x, y^{\prime}}}{\theta_{x, y}}=\frac{s(x)^{-1}+s\left(y^{\prime}\right)^{-1}}{s(x)^{-1}+s(y)^{-1}} \geq \frac{s\left(y^{\prime}\right)^{-1}}{s(y)^{-1}} \geq \frac{s(y)}{s(y)+1} \geq 1 / 2 .
$$


The first inequality follows from the observation $\frac{c+a}{c+b} \geq \frac{a}{b}$ whenever $a \leq b$ and $c \geq 0$. Since $\ell=2\left\lceil C_{\varepsilon} \sqrt{n}\right\rceil, s(y) \geq \ell-\frac{\sigma \ell}{16 C_{\varepsilon}} \geq 1$, yielding the final inequality $\mathrm{u}$

Claim 2.3.3. For $(x, y) \in \Pi$,

$$
\frac{\operatorname{Pr}\left[\mathcal{E}_{x, E(y)}\right]}{\operatorname{Pr}\left[\mathcal{E}_{x, y}\right]}=\Omega\left(\frac{\sigma}{\sqrt{n}}\right)
$$

Proof. Combining Claim 2.3.1 and Claim 2.3.2,

$$
\frac{\operatorname{Pr}\left[\mathcal{E}_{x, E(y)}\right]}{\operatorname{Pr}\left[\mathcal{E}_{x, y}\right]}=\frac{\theta_{x, E(y)}\left|\mathcal{P}_{x, E(y)}\right|}{\theta_{x, y}\left|\mathcal{P}_{x, y}\right|} \geq \frac{\left|\mathcal{P}_{x, E(y)}\right|}{2\left|\mathcal{P}_{x, y}\right|}
$$

We know exactly what both the numbers in the RHS are. Say $|x|=t$ and $|y|=t+u$. Note $u \geq \sigma \ell / 32 C_{\varepsilon}-1$ and $|E(y)|=|y|+1$. Then,

$$
\left|\mathcal{P}_{x, y}\right|=t ! u !(n-u-t) ! \quad \text { and } \quad\left|\mathcal{P}_{x, E(y)}\right|=t !(u+1) !(n-u-t-1) !
$$

Plugging in (2.6),

$$
\frac{\operatorname{Pr}\left[\mathcal{E}_{x, E(y)}\right]}{\operatorname{Pr}\left[\mathcal{E}_{x, y}\right]} \geq \frac{u+1}{2(n-u-t)}
$$

The denominator is $\Theta(n)$ since $n / 2-C_{\varepsilon} \sqrt{n} \leq|y| \leq n / 2+C_{\varepsilon} \sqrt{n}$, and $C_{\varepsilon} \leq 2 \sqrt{\ln n}$. The numerator is at least $\sigma \ell / 32 C_{\varepsilon}=\Omega(\sigma \sqrt{n})$, completing the proof.

Going back to (2.3),

$$
\begin{aligned}
\operatorname{Pr}[\text { success }] & \geq \sum_{(x, y) \in \Pi} \operatorname{Pr}\left[\mathcal{E}_{x, y}\right] \cdot \frac{\operatorname{Pr}\left[\mathcal{E}_{x, E(y)}\right]}{\operatorname{Pr}\left[\mathcal{E}_{x, y}\right]} \\
& =\Omega\left(\frac{\sigma}{\sqrt{n}} \cdot \sum_{(x, y) \in \Pi} \operatorname{Pr}\left[\mathcal{E}_{x, y}\right]\right)
\end{aligned}
$$

Now for the punchline. Color all points in $B_{1}$ blue. By the choice of parameters, $\left|B_{1}\right|=\sigma 2^{n}$. Lemma 2.2 tells us that the probability of path-tester $(\sigma)$ sampling a pair $(x, y)$ such that both points are blue is $\Omega\left(\sigma^{2} / \ln (1 / \varepsilon)\right)$. This is the event that $x \prec y$ (or $y \prec x),\|y-x\|_{1} \geq \frac{\sigma \ell}{32 C_{\varepsilon}}-1$, and $x, y \in B_{1}$. The probability of this event is exactly twice $\sum_{(x, y) \in \Pi} \operatorname{Pr}\left[\mathcal{E}_{x, y}\right]$. Hence, the probability of success is $\Omega\left(\frac{\sigma^{3}}{\sqrt{n} \ln (1 / \varepsilon)}\right)$.

口

2.3. Wrapping it up with the dichotomy. We state the directed variant of Margulis' theorem. We actually prove a slightly stronger dichotomy theorem between the total number of violated edges and largest matching of violated edges. We let $\Phi_{f}^{+}$be the number of violated edges divided by $2^{n-1}$ (think of this as the "violation influence"). We set $\Gamma_{f}^{+}$to be the size of the largest matching of violated edges in the middle layers divided by $2^{n}$.

THEOREM 2.4. For any function $f$ that is $\varepsilon$-far from monotone, $\Phi_{f}^{+} \cdot \Gamma_{f}^{+} \geq \frac{\varepsilon^{2}}{32}$. 
Proof. Recall, since $f$ is $\varepsilon$-far from monotonicity, any maximal matching $M$ of violated pairs (not edges) must have cardinality $|M| \geq \varepsilon 2^{n-1}$. For any such matching $M$ of violated pairs, define the average length of $M$ to be the quantity

$$
|M|^{-1} \sum_{(x, y) \in M}\|y-x\|_{1}
$$

Choose $M$ to be a maximum cardinality matching of violated pairs with the smallest average length, denoted by $r$.

LEMma 2.5. If the average length of $M$ is $r$, then $\Gamma_{f}^{+} \geq \frac{\varepsilon}{32 r}$.

That is, there exists a matching $E$ of violated edges all lying in the middle layers of the hypercube with size at least $\frac{\varepsilon 2^{n}}{32 r}$.

Proof. Deferred to $\S 2.4$.

Lemma 2.6. If the average length of $M$ is $r$, then $\Phi_{f}^{+} \geq r \varepsilon$.

That is, there are at least $r \varepsilon 2^{n-1}$ violated edges.

Proof. Deferred to $\S 2.5$. .

The proof of the theorem follows from the above two lemmas.

It is now routine to prove Theorem 1.1 and Theorem 1.2.

Proof of Theorem 1.1: Set $s=n^{1 / 8} \varepsilon^{3 / 2}$ and $\sigma=n^{-1 / 8} \varepsilon^{1 / 2} / 32$. We will argue that either the edge tester or path-tester $(\sigma)$ has a success probability of $\Omega\left(n^{-7 / 8} \varepsilon^{3 / 2} \ln ^{-1}(1 / \varepsilon)\right)$.

The success probability of the edge tester is exactly $\Phi_{f}^{+} / n$. If $\Phi_{f}^{+} \geq s$, then the edge tester succeeds with the desired probability. So let us assume that $\Phi_{f}^{+}<s$. By Theorem 2.4, $\Gamma_{f}^{+} \geq \frac{\varepsilon^{2}}{32 s}=\sigma$. Therefore, there exists a matching of violated edges of size $\sigma 2^{n}$, all of them lying in the middle layers. We apply Lemma 2.3 and bound the success probability of path-tester $(\sigma)$ by $\Omega\left(\frac{\sigma^{3}}{\sqrt{n} \ln (1 / \varepsilon)}\right)=\Omega\left(n^{-7 / 8} \varepsilon^{3 / 2} \ln ^{-1}(1 / \varepsilon)\right)$.

Proof of Theorem 1.2: Note that $\Phi_{f}^{+} \leq \mathbf{I}(f)$. From Theorem 2.4, $\Gamma_{f}^{+} \geq \frac{\varepsilon^{2}}{32 \mathbf{I}(f)}$. If we set $\sigma$ to be this lower bound, then path-tester $(\sigma)$ succeeds with probability $\Omega\left(n^{-1 / 2} \varepsilon^{6} \mathbf{I}^{-3}(f) \ln ^{-1}(1 / \varepsilon)\right)$. We do not need the edge tester for these functions.

\subsection{Proof of Lemma 2.5.}

We first state the routing theorem of Lehman and Ron. Recall that $L_{i}$ was defined as the set of points of the hypercube with exactly $i$ ones.

Theorem 2.7 (Lehman-Ron [11]). Let $S \subseteq L_{i}$ and $R \subseteq L_{j}$ with $|S|=|R|=m$ and $i<j$. Furthermore, suppose there is a bijection $\phi: S \mapsto R$ such that $x \succ \phi(x), \forall x \in$ $S$, that is, $(x, \phi(x))$ are ancestor-descendants. Then there exists $m$ vertex disjoint directed paths from the set $S$ to $R$.

For convenience, we represent the matching through ordered pairs, so if $(x, y) \in M$, then $x \prec y$ (and $f(x)=1, f(y)=0$ ). Recall $M$ is a maximum cardinality matching in the violated graph with the smallest average length. Among such matchings, let 
$M$ actually be one maximizing

$$
\Psi(M):=\sum_{(x, y) \in M}\|x-y\|_{1}^{2}
$$

We prove a structural claim regarding $M$. Two pairs $(x, y)$ and $\left(x^{\prime}, y^{\prime}\right)$ cross if (a) there exists a $z$ such that $x \prec z \prec y$ and $x^{\prime} \prec z \prec y^{\prime}$ and (b) the intervals $[|x|,|y|]$ and $\left[\left|x^{\prime}\right|,\left|y^{\prime}\right|\right]$ strictly cross, meaning that neither interval contains the other. Note that the interval $[|x|,|y|]$ and $\left[\left|x^{\prime}\right|,\left|y^{\prime}\right|\right]$ are ranges of integers. (By (a), the intervals $[|x|,|y|]$ and $\left[\left|x^{\prime}\right|,\left|y^{\prime}\right|\right]$ must intersect.) Recall $|x|$ is the number of ones in $x$.

Claim 2.7.1. There are no crossing pairs in $M$.

Proof. Suppose $(x, y)$ and $\left(x^{\prime}, y^{\prime}\right)$ cross. Consider $M^{\prime}$ formed by deleting these pairs from $M$ and adding $\left(x, y^{\prime}\right)$ and $\left(x^{\prime}, y\right)$. These are valid violations due to the presence of the vertex $z$. Furthermore, observe that $\Psi\left(M^{\prime}\right)>\Psi(M)$ since the sum of squares of a pair of numbers having a fixed sum increases as the maximum (of the pair) increases.

\section{口}

For every two levels $i<j$ of the hypercube, let $M_{i, j} \subseteq M$ be the subset of pairs with endpoints in the level sets $L_{i}$ and $L_{j}$. Apply Theorem 2.7 to get a collection of $\left|M_{i, j}\right|$ vertex disjoint paths. Each of these vertex disjoint paths contain at least one violated edge, and let $F_{i, j}$ be the set of these edges. Note that $F_{i, j}$ forms a matching. Consider the multiset $F$ formed by the union of $F_{i, j}$ over the set $\left\{(i, j): i, j \in I_{\ell}, i<\right.$ $j, j-i \leq 2 r\}$. Note that $F$ may contain more than one copy of the same edge. Also note that all edges of $F$ lie in the middle layers.

Claim 2.7.2. $|F| \geq|M| / 4$.

Proof. Note that $|F|=\sum_{(i, j): i, j \in I_{\ell}, i<j, j-i \leq 2 r}\left|M_{i, j}\right|$. Since the matching $M$ has average length $r$, by Markov's inequality, at least $|M| / 2$ of these pairs have length at most $2 r$. Furthermore, from Prop. 2.1 we get that at most $\varepsilon^{5} 2^{n} \leq|M| / 4$ pairs in $M$ have endpoints not in the middle layer. Looking at the remainder, we get $\sum_{(i, j): i, j \in I_{\ell}, i<j, j-i \leq 2 r}\left|M_{i, j}\right| \geq|M| / 4$.

ClaIm 2.7.3. No point $z \in\{0,1\}^{n}$ has more than $2 r$ edges of $F$ incident on it.

Proof. Pick a vertex $z$, and pick any two edges $f_{1}$ and $f_{2}$ of $F$ incident on it. Since each $F_{i, j}$ is a matching, these must lie in different $F_{i, j}$ 's. Suppose they are $F_{i, j}$ and $F_{a, b}$, where $i$ could be $a$ and $j$ could be $b$, but not both together. Note that $i \leq|z| \leq j$ and $a \leq|z| \leq b$. We claim that $[i, j]$ and $[a, b]$ cannot cross, and therefore one must strictly lie in the other. There can be at most $2 r$ intervals containing $|z|$ satisfying such containment relationships. Thus, there can be most $2 r$ edges of $F$ incident on $z$.

We claim that $[i, j]$ and $[a, b]$ cannot cross. To see this, consider the pairs in $M_{i, j}$, and let them be $\left(x_{1}, y_{1}\right),\left(x_{2}, y_{2}\right), \ldots,\left(x_{k}, y_{k}\right)$. Note that Theorem 2.7 implies $k$ vertex disjoint paths containing all these vertices. Hence, there is some permutation $\pi$ such that for each $i \in[k]$, there is a path from $x_{i}$ to $y_{\pi(i)}$. Let $M_{i, j}^{\prime}=\left\{\left(x_{i}, y_{\pi(i)}\right)\right\}$. Similarly, define $M_{a, b}^{\prime}$. Let $M^{\prime}$ be the matching where $M_{i, j}$ and $M_{a, b}$ are replaced by $M_{i, j}^{\prime}$ and $M_{a, b}^{\prime}$, respectively, and all other pairs remain. Note that $M^{\prime}$ has the same average length, same cardinality and $\Phi\left(M^{\prime}\right)=\Phi(M)$. But now, we have a pair $(x, y) \in M_{i, j}^{\prime}$ such that $x \prec z \prec y$, and a pair $\left(x^{\prime}, y^{\prime}\right) \in M_{a, b}^{\prime}$ such that $x^{\prime} \prec z \prec y^{\prime}$. 
This is because $z$ is incident to an edge in both $F_{i, j}$ and $F_{a, b}$. If $[i, j]$ and $[a, b]$ cross, then $(x, y)$ and $\left(x^{\prime}, y^{\prime}\right)$ cross. Since $M^{\prime}$ maximizes the potential $\Phi$, Claim 2.7.1 is contradicted.

Since the multigraph induced by $F$ has maximum degree $2 r$, there exists a matching $E \subseteq F$ of size $|E| \geq|F| / 4 r$. This can be obtained by picking an edge in $E$ arbitrarily and deleting all edges incident to any of its endpoints from $F$. For every edge added to $E$ there are at most $4 r$ edges deleted from $F$. Therefore, following Claim 2.7.2, we get a matching $E$ of violated edges of size $\geq|M| / 16 r \geq \varepsilon 2^{n-5} / r$. Furthermore, they all lie in the middle layers. This completes the proof of Lemma 2.5.

\subsection{Proof of Lemma 2.6.}

Let $M_{i}$ be the set of pairs in $M$ that cross dimension $i$, that is, $M_{i}:=\{(x, y) \in M$ : $\left.x_{i}=1, y_{i}=0\right\}$. Lemma 2.6 follows from following theorem.

TheOREM 2.8. For all $i$, the number of violated edges across dimension $i$ is at least $\left|M_{i}\right|$.

Note that $\sum_{i}\left|M_{i}\right|=\sum_{(x, y) \in M}\|y-x\|_{1}=r|M|$, since a pair $(x, y)$ appears in precisely $\|y-x\|_{1}$ different $M_{i}$ 's. Theorem 2.8 implies that the number of violated edges is at least $\sum_{i}\left|M_{i}\right|$. Lemma 2.6 follows because $|M| \geq \varepsilon 2^{n-1}$.

We now prove Theorem 2.8.

Proof. This requires setting up some of the machinery of [4]. Let $H$ be the perfect matching of the hypercube formed by the edges crossing the $i$ th dimension. Let $X$ be the endpoints of $M_{i}$. For all $x \in X$, we now define a sequence $\mathbf{S}_{x}$. In what follows, we use the shorthand $M(v)$ and $H(v)$ to denote the partners of $v$ in the matchings $M$ and $H$, respectively. The first term of the sequence, $\mathbf{S}_{x}(0)$, is $x$. For even $i, \mathbf{S}_{x}(i+1)=H\left(\mathbf{S}_{x}(i)\right)$. For odd $i$, if $\mathbf{S}_{x}(i) \in X$, or is $M$-unmatched, then $\mathbf{S}_{x}$ terminates. Otherwise, $\mathbf{S}_{x}(i+1)=M\left(\mathbf{S}_{x}(i)\right)$. The best way to think about $\mathbf{S}_{x}$ is via alternating paths and cycles formed by the matchings $M$ and $H$. We start at $x$ and take the $H$-edge along the alternating path. We alternate between $H$ and $M$ edges till we reach an endpoint of the alternating path or another vertex in $X$. Thus, each $\mathbf{S}_{x}$ terminates. It is not hard to see that if $\mathbf{S}_{x}$ ends at $y \in X$, then $\mathbf{S}_{y}$ is just $\mathbf{S}_{x}$ in reverse. Also note that $\mathbf{S}_{x}$ and $\mathbf{S}_{y}$ are disjoint unless $y$ terminates $\mathbf{S}_{x}$, for otherwise $\mathbf{S}_{x}$ would've terminated earlier. Therefore the number of sequences is at least $|X| / 2=\left|M_{i}\right|$. The theorem is a consequence of the following lemma.

Lemma 2.9. For all $x, \mathbf{S}_{x}$ contains a violated edge in $H$.

Proof. We will prove this through contradiction, and will henceforth assume that (for some $x$ ), $\mathbf{S}_{x}$ has no violated edge in $H$. We show that $\mathbf{S}_{x}$ cannot terminate, completing the contradiction. For brevity, let us use $s_{j}$ to denote $\mathbf{S}_{x}(j)$. Let $(x, y)$ be the pair in $M_{i}$. We use $s_{-1}$ to denote $y$. Wlog, assume $x \succ y$, thus $x_{i}=1$ and $y_{i}=0$. Also $f(y)=1$ and $f(x)=0$ since the pair is a violation.

Let $\mathrm{B}_{b}(b=\{0,1\})$ be the $n-1$ dimensional hypercube where $i$ th coordinate is $b$. We will use $d\left(x, x^{\prime}\right)$ for the Hamming distance between two points $x$ and $x^{\prime}$. We have the following simple claim.

Claim 2.9.1. Let $j \geq 0$ be an index and suppose $s_{j}$ exists. 
If $j \equiv 0(\bmod 4)$,

- $f\left(s_{j}\right)=0$.

If $j \equiv 1(\bmod 4)$,

- $f\left(s_{j}\right)=0$.

- $s_{j} \in \mathrm{B}_{1}$.

- $s_{j} \in \mathrm{B}_{0}$.

If $j \equiv 2(\bmod 4)$,

- $f\left(s_{j}\right)=1$.

- $s_{j} \in \mathrm{B}_{0}$.

If $j \equiv 3(\bmod 4)$,

- $f\left(s_{j}\right)=1$.

- $s_{j} \in \mathrm{B}_{1}$.

Proof. We prove by induction on $j$. For the base case, $s_{0}=x$, and $f(x)=0$, $s_{0} \in \mathrm{B}_{1}$. Consider $j \equiv 0(\bmod 4), j \geq 1$. By the induction hypothesis, $f\left(s_{j-1}\right)=1$ and $s_{j-1} \in \mathrm{B}_{1}$. Since $s_{j}=M\left(s_{j-1}\right)$ and thus $\left(s_{j-1}, s_{j}\right)$ is a violation, $f\left(s_{j}\right)=0$ and $s_{j} \in \mathrm{B}_{1}$. Consider $j \equiv 1(\bmod 4)$. By the induction hypothesis, $f\left(s_{j-1}\right)=0$ and $s_{j-1} \in \mathrm{B}_{1}$. Since $s_{j}=H\left(s_{j-1}\right)$ and $\left(s_{j-1}, s_{j}\right)$ is not a violation by assumption that no $H$-edge is a violation, $f\left(s_{j}\right)=0$ and $s_{j} \in \mathrm{B}_{0}$. The remaining cases are analogous.

口

Claim 2.9.2. Let $j \geq 0$ be even. Then $\left(s_{j}, s_{j+3}\right)$ is a violation and $d\left(s_{j}, s_{j+3}\right)=$ $d\left(s_{j+1}, s_{j+2}\right)$. Also, the pair $\left(y, s_{1}\right)$ is a violation and $d\left(y, s_{1}\right)=d\left(y, s_{0}\right)-1$.

Proof. Suppose $j \equiv 2(\bmod 4)$. From Claim 2.9.1, $f\left(s_{j}\right)=1$ and $f\left(s_{j+3}\right)=0$. The claim also implies $f\left(s_{j+1}\right)=1$ and since $\left(s_{j+1}, s_{j+2}\right) \in M, s_{j+2} \succ s_{j+1}$. Furthermore, both $s_{j+1}, s_{j+2} \in \mathrm{B}_{1}$. Since $s_{j}=H\left(s_{j+1}\right)$ and $s_{j+3}=H\left(s_{j+2}\right)$, we get (a) $s_{j+3} \succ s_{j}$ as well, implying $\left(s_{j}, s_{j+3}\right)$ is a violation, and (b) $d\left(s_{j}, s_{j+3}\right)=d\left(s_{j+1}, s_{j+2}\right)$. The case $j \equiv 0(\bmod 4)$ is analogous.

Observe that $\left(y, s_{0}\right)$ is a violation where $y \in \mathrm{B}_{0}$ and $s_{0} \in \mathrm{B}_{1}$. Since $s_{1}=H\left(s_{0}\right), s_{1}$ has the same coordinates as $s_{0}$ except for the $i$ th one. Also, $f\left(s_{1}\right)=0$. Therefore, $\left(y, s_{1}\right)$ is a violation and $d\left(y, s_{1}\right)=d\left(y, s_{0}\right)-1$.

We argue that $\mathbf{S}_{x}$ cannot terminate, by showing for any odd $j, s_{j+1}$ must exists. (This is trivially true for even $j$, since $H$ is a perfect matching.) We focus on $j \equiv 1(\bmod 4)$ (the case $j \equiv 3(\bmod 4)$ is analogous). Because $f\left(s_{j}\right)=0$ and $s_{j} \in \mathrm{B}_{0}, s_{j}$ cannot participate in a pair in $M_{i}$. Hence, $s_{j} \notin X$. Suppose $s_{j}$ was unmatched. Consider the following set of pairs in $M: A=\left\{\left(s_{k}, s_{k+1}\right) \mid k\right.$ odd, $\left.-1 \leq k \leq j-2\right\}$. Suppose we replaced these pairs in $M$ by $B=\left\{\left(y, s_{1}\right)\right\} \cup\left\{\left(s_{k}, s_{k+3}\right) \mid k\right.$ even, $\left.0 \leq k \leq j-3\right\}$. Note that $|A|=\lceil j / 2\rceil=|B|$. Also, by Claim 2.9.2

$$
d\left(y, s_{0}\right)+\sum_{\substack{k=1 \\ k \text { odd }}}^{j-2} d\left(s_{k}, s_{k+1}\right)=d\left(y, s_{1}\right)-1+\sum_{\substack{k=0 \\ k \text { even }}}^{j-3} d\left(s_{k}, s_{k+3}\right)
$$

This means that replacing $A$ and inserting $B$ (in $M$ ) leads to a violation matching of the same size with a smaller Hamming distance. This violates the property that $M$ has minimum average Hamming distance, and therefore, the sequence $\mathbf{S}_{x}$ cannot terminate. This cannot occur, and therefore, every $\mathbf{S}_{x}$ must contain a violated edge in $H$. This ends the proof of Lemma 2.9 .

3. Conclusion. In this paper, we make progress on the question of testing monotonicity of Boolean functions over the hypercube. Our approach falls short of the known $\Omega(\sqrt{n})$ lower bound for one-sided, non-adaptive testers. Nevertheless, we believe the path tester (alone) is a $O(\sqrt{n})$-query monotonicity tester for Boolean functions. A possible approach is suggested by Theorem 1.2. Can we perform a different analysis (or even design a different algorithm) for high average sensitivity functions?

4. Acknowledgements. We thank Cyrus Rashtchian and Naomi Kirshner for pointing out a mistake in an older version. 
Sandia National Laboratories is a multi-program laboratory managed and operated by Sandia Corporation, a wholly owned subsidiary of Lockheed Martin Corporation, for the U.S. Department of Energy's National Nuclear Security Administration under contract DE-AC04-94AL85000. CS is grateful for the support received from the Early Career LDRD program at Sandia National Laboratories.

\section{REFERENCES}

[1] N. Ailon, B. Chazelle, S. Comandur, and D. Liu, Estimating the distance to a monotone function, Random Structures and Algorithms 31 (2006), no. 3, 1704-1711. 1

[2] E. Blais, J. Brody, and K. Matulef, Property testing lower bounds via communication complexity, Computational Complexity 21 (2012), no. 2, 311-358. 1

[3] J. Briët, S. Chakraborty, D. García-Soriano, and A. Matsliah, Monotonicity testing and shortest-path routing on the cube, Combinatorica 32 (2012), no. 1, 35-53. 1, 2

[4] D. Chakrabarty and C. Seshadhri, Optimal bounds for monotonicity and Lipschitz testing over the hypercube, Tech. Report TR12-030, ECCC, April 2012. 1, 3, 11

[5] Y. Dodis, O. Goldreich, E. Lehman, S. Raskhodnikova, D. Ron, and A. Samorodnitsky, Improved testing algorithms for monotonicity, Proceedings of the 3rd International Workshop on Randomization and Approximation Techniques in Computer Science (RANDOM) (1999), 97-108. 1, 2

[6] F. Ergun, S. Kannan, R. Kumar, R. Rubinfeld, and M. Viswanathan, Spot-checkers, Journal of Computer Systems and Sciences (JCSS) 60 (2000), no. 3, 717-751. 1

[7] E. Fischer, E. Lehman, I. Newman, S. Raskhodnikova, and R. Rubinfeld, Monotonicity testing over general poset domains, Proceedings of the 34th Annual ACM Symposium on the Theory of Computing (STOC) (2002), 474-483. 1, 2, 4

[8] O. Goldreich, S. Goldwasser, E. Lehman, D. Ron, and A. Samordinsky, Testing monotonicity, Combinatorica 20 (2000), 301-337. 1, 3

[9] S. Halevy and E. Kushilevitz, Testing monotonicity over graph products, Random Structures and Algorithms 33 (2008), no. 1, 44-67. 1

[10] K. H. Harper, Optimal numberings and isoperimetric problems on graphs, Journal of Combinatorial Theory 1 (1966), 385-393. 3

[11] E. Lehman and D. Ron, On disjoint chains of subsets, Journal of Combinatorial Theory, Series A 94 (2001), no. 2, 399-404. 1, 3, 9

[12] G. A. Margulis, Probabilistic characteristics of graphs with large connectivity, Problemy Peredachi Informatsii 10 (1974), no. 2, 101-108. 3

[13] M. Parnas, D. Ron, and R. Rubinfeld, Tolerant property testing and distance approximation, Journal of Computer and System Sciences 6 (2006), no. 72, 1012-1042. 1

[14] D. Ron, R. Rubinfeld, S. Safra, and O. Weinstein, Approximating the Influence of Monotone Boolean Functions in $O(\sqrt{n})$ Query Complexity., Proceedings of the 15th International Workshop on Randomization and Approximation Techniques in Computer Science (RANDOM), 2011. 2 